
\title{
CONTABILIDAD, UNIVERSIDAD Y DESARROLLO SOSTENIBLE
}

\section{ACCOUNTING, UNIVERSITY, AND SUSTAINABLE DEVELOPIENT}

MARÍA BEATRIZ MAZA

Facultad de Ciencias Económicas y de Administración. Universidad Nacional de Catamarca. Argentina.

(iD) $0000-0002-9513-4479$

\mariabeatrizmaza@gmail.com

\section{NANNA DAYAN LASCANC}

Facultad de Ciencias Económicas y de Administración. Universidad Nacional de Catamarca. Argentina.

(iD) 0000-0003-0325-2492

$\bowtie$ dayan.lascano@gmail.com

\section{JORGE OMAR ZAFE}

Facultad de Ciencias Económicas y de Administración

Universidad Nacional de Catamarca. Argentina.

(iD) 0000-0001-9332-5047

凶jorgezafe@gmail.com

COLABORADORA:

MARÍA YANET SALAS CEDRÓN Facultad de Ciencias Económicas y de Administración. Universidad Nacional de Catamarca. Argentina.

iD 0000-0003-1379-1414

$\triangle$ yanetsalas5@gmail.com

\section{PALABRAS CLAVE}

contabilidad social y ambiental - responsabilidad social universitaria - desarrollo sostenible.

\section{RESUMEN}

La responsabilidad social es un tema que ha adquirido relevancia en el contexto académico. La universidad es ella misma una realidad social y su actividad tiene un inevitable impacto en su entorno. El propósito de este estudio es analizar cómo la disciplina contable puede informar sobre la forma de administrar el impacto social, económico y ambiental de las actividades universitarias: académicas, investigación, extensión y gestión. También, establecer e informar sobre el aporte a la consecución del desarrollo sostenible y a los objetivos de desarrollo sostenible (ODS).

Se contextualiza la base teórica desde el modelo de gestión de impactos de François Vallaeys. Esta investigación siguió un enfoque cuali-cuantitativo, con recopilación de datos a través de encuestas, observación y documentación de tipo no experimental con carácter descriptivo.

El Balance Social para la Facultad de Ciencias Económicas y Administración de la Universidad Nacional de Catamarca que se presenta en este trabajo se realizó siguiendo un modelo elaborado por el mismo grupo de investigación y ya presentado en anteriores presentaciones. El modelo se elaboróadaptando a la organización universitaria las previsiones de la Resolución Técnica №6 y sus modificaciones de la Federación Argentina de Consejos Profesionales en Ciencias Económicas

Asimismo, se incluyó una relación de las actividades universitarias con los Objetivos de Desarrollo Sostenible incluidos en la Agenda 2030.

Los resultados muestran evidencia de la posibilidad de realizar Balances Sociales para las Universidades Nacionales empleando el modelo propuesto como manera de mantener informados de modo más transparente a los diferentes grupos de interés -que se definan en cada organización universitaria- de los impactos de las acciones docentes, de investigación, extensión y gestión realizados en un lapso predeterminado. Asimismo, el Balance Social confeccionado resulta ser una excelente herramienta de gestión por cuanto los indicadores planteados para las diferentes actividades están relacionados con los estándares para la acreditación de las carreras de Contador Público argentinas.

La Responsabilidad Social Universitaria (RSU) no se trata solo de decirse responsables, se trata de serlo, lo cual implica algo más que sólo hablar de ello o hacer uno o dos cambios en el intento; se deben Ilevar adelante acciones consecutivas, medibles, que buscan informar a los grupos de interés, corregir y/o mejorar la gestión.

\section{ABSTRACT}

Social responsibility is a topic that has gained relevance in the academic context. The university is a social reality in itself, and its activity has an inevitable impact on its environment. This paper aims to analyse how the accounting discipline can inform about how to manage the social, economic, and environmental impact of academic, research, extension, and management university activities. It also aims to establish and report on the contribution to the achievement of sustainable development and the Sustainable Development Goals (SDGs). The theoretical basis is contextualised from François Vallaeys' impact management model. This research follows a qualitative-quantitative approach, with data collection through surveys, observation and descriptive non-experimental documentation.

The Social Balance Sheet for the School of Economic Sciences and Administration of the National University of Catamarca presented in this paper was carried out following a model developed by the same research group and already introduced in previous presentations. The model was elaborated by adapting to the university organisation the provisions of the Technical Resolution No. 36 as amended of the Argentine Federation of Professional Councils in Economic Sciences.

A relationship between university activities and the Sustainable Development Goals included in the 2030 Agenda was also included.

The results show evidence of the possibility of carrying out Social Balance Sheets for National Universities by using the proposed model as a way to keep the different shareholders — as defined in each university organisation - informed of the impacts of the teaching, research, extension, and management actions carried out in a predetermined period of time in a more transparent way. Moreover, the Social Balance Sheet is an excellent management tool because the indicators proposed for the different activities are related to the standards for the accreditation of Argentinean Public Accountant degrees.

University Social Responsibility (USR) is not only about claiming to be responsible, but it is about being responsible, which implies more than just talking about it or making one or two changes in an attempt to be responsible. Consecutive and measurable actions must be taken to inform shareholders, correct, or improve management.
Recibido 23 de agosto, 2021 Aprobado 15 de septiembre, 2021

CÓDIGO JEL 056 - M14
Revista Proyecciones Año 2021 № 15 DOI: https://doi.org/10.24215/26185474e016 ISSN 2618-5474 https://revistas.unlp.edu.ar/proyecciones
Entidad editora

instituto de Investigaciones y Estudios Contables Facultad de Ciencias Económicas - UNLP. 


\section{INTRODUCCIÓN}

Este trabajo continúa con el proceso de investigación del Proyecto de Investigación Contabilidad y Universidad dirigido por la profesora María Beatriz Maza, con vigencia desde el 01/01/2016 al 31/12/2019 y que por efectos de la pandemia se prorrogó hasta el 2020. La investigación se reforzó con el proyecto tipo B de la Facultad de Ciencias Económicas y Administración (FCEyA) denominado "Modelo de Indicadores para un Balance de Responsabilidad Social Universitaria".

La sociedad contemporánea es cada día más consciente de que el deterioro del medio ambiente, el consumo exagerado de los recursos naturales, el trabajo infantil, la contaminación, la discriminación, el uso inadecuado de los factores de la producción, las publicidades engañosas, entre otros factores, han generado un movimiento que afecta a todos en su condición de seres humanos. Actualmente, como consecuencia de la pandemia que se vive en todo el mundo, la toma de consciencia es aún mayor.

Por ello se observa día a día el nacimiento de diferentes organizaciones que se propusieron lograr mejoras sustantivas mediante el requerimiento a las empresas y a las organizaciones en general para que asuman mayores compromisos ante sus clientes y ante la sociedad para lograr un desarrollo sostenible. En todo el mundo la revelación de lo social y lo ambiental está adquiriendo fuerza creciente. Nacen así los fundamentos de las memorias de responsabilidad social empresaria o corporativa, de los informes de sostenibilidad 0 sustentabilidad y de balance social.

Argentina no está aislada del resto del mundo, nuevos actores y actitudes irrumpen en nuestra sociedad. Las organizaciones no gubernamentales, la inmensa demanda de transparencia, rendición de cuentas y reducción de los costos de la política se suman a la existencia del voluntariado social y corporativo. Los reclamos legales y sociales de revelación de políticas para el desarrollo sustentable, así como las estrategias respecto de la gestión de los recursos humanos son temas que adquieren relevancia.

El Balance Social es el informe a través del cual los segmentos de la Contabilidad Social y Ambiental permiten a las organizaciones mostrar a la sociedad la manera en que asumen su responsabilidad social. El mundo empresarial ha acogido estos conceptos desde hace algún tiempo y ha evolucionado durante décadas en sus aspectos teóricos. Los Contadores Públicos, en su condición de expertos en Contabilidad, han aportado a la evolución de estos conocimientos.

A su vez, los universitarios están siendo testigos de reflexiones mucho más recientes sobre la Responsabilidad Social Universitaria (RSU). Parece útil, entonces, utilizar el conocimiento y experiencia adquirido en el mundo empresarial para realizar aportes para la evolución de la RSU, entendiendo siempre que la naturaleza de la Universidad no es ni debe ser similar a la de las empresas.

\section{EL PROBLEMA PLANTEADO}

Con el fin de aportar desde las ciencias económicas a los avances mundiales de la Responsabilidad Social Empresarial (RSE) y a los avances de la contabilidad en el/los segmento/s de Contabilidad Social y Ambiental como herramientas utilizables para transparentar la RSU, se han formulado los siguientes interrogantes:

¿Es posible realizar un Balance Social Universitario, siguiendo alguno de los modelos existentes actualmente?

¿Cuáles serían los grupos de interés que resultarían beneficiados con la confección del Balance Social Universitario?

¿Cuál sería el modelo más adecuado para su elaboración?

¿Qué beneficios obtendrían los grupos de interés identificados?

\section{OBJETIVOS DE LA INVESTIGACIÓN}

El proyecto de investigación de la Facultad de Ciencias Económicas y de Administración de la Universidad Nacional de Catamarca (FCEyA-UNCa) persiguió como objetivos generales:

Elaborar un concepto de responsabilidad social universitaria a partir de análisis y comparación de diferentes concepciones de responsabilidad social empresaria y la mirada de diferentes actores universitarios;

Elaborar un modelo de Balance Social Universitario;

Elaborar el Balance Social de la FCEyA-UNCa.

Los avances del proyecto se fueron compartiendo en diferentes encuentros de investigación. Hoy se pretende compartir el Balance Social 2018 realizado como finalización del proyecto.

\section{JUSTIFICACIÓN DEL ABORDAJE}

Las Universidades son organizaciones y, por lo tanto, corresponde por definición pensar en cómo se está asumiendo la responsabilidad social que les cabe como tales. Además, estas particulares organizaciones tienen como objetivo prioritario formar profesionales que habrán de prestar servicios a empresas, ciuda- 
danos que habrán de promover los derechos humanos y futuros funcionarios que tendrán a su cargo decisiones relacionadas con el bien común de nuestras comunidades (Vallaeys, 2006).

Es por ello que se debe colocar la responsabilidad social universitaria (RSU) en la agenda prioritaria de las universidades. Sin embargo, no es mucho lo que se ha avanzado en este sentido. Desde las ciencias económicas se pueden realizar aportes utilizando la RSU y, a su vez, diferenciándola de la responsabilidad social empresarial (RSE).

Así como los estados contables de una organización deben reflejar de forma transparente la situación económica, patrimonial y financiera de esta, los reportes sociales lo deben hacer en relación a la realidad social, ambiental y económica. Para lograr transparencia y credibilidad en los reportes, estos deben ser elaborados con un compromiso de las organizaciones sobre valores sociales que resultan fundamentales para obtener credibilidad de largo plazo

El desarrollo del concepto de RSE ha obligado a las empresas a definir reportes sociales que certifiquen las actividades de responsabilidad social realizadas. Dentro de los reportes se pueden encontrar los "Códigos de Conducta", donde se comunican los valores y las prácticas comerciales de la empresa; las "Memorias Sociales", donde se describe la actuación socialmente responsable de la empresa, y los "Balances Sociales", donde se cuantifican las prácticas responsables de la empresa. Su presentación a la sociedad y a las partes relacionadas con la empresa da un mayor sentido de transparencia en la gestión y sirve de ejemplo a otras empresas contribuyendo a aumentar la difusión de la importancia de la RSE.

Por ello, usar lo aprendido en el ambiente empresarial para avanzar en una definición de la responsabilidad social universitaria, su comprensión y acción y la consecuente elaboración de su Balance Social, ayudará a mejorar estratégicamente la gestión y brindará información a los grupos de interés que deberán definirse para la organización en análisis.

\section{EL BALANCE SOCIAL UNIVERSITARIO Y LOS OBJETIVOS DEL DESARROLLO SOSTENIBLE}

Se había definido responsabilidad social universitaria como "las obligaciones de los gestores universitarios para impulsar políticas institucionales, para tomar decisiones o para seguir líneas de acción que son deseables en términos de los objetivos y valores de la sociedad" (Gaete Quezada, 2017, p. 136).
El modelo de Balance Social Universitario (BSU), confeccionado adaptando el previsto por las normas contables profesionales argentinas, permite mostrar cómo impactan en la sociedad las diferentes acciones universitarias impulsadas o no por los gestores universitarios. Las preguntas entonces fueron ¿las acciones realizadas son las deseables en términos de objetivos y valores de la sociedad? y ¿cuáles son los objetivos y valores sociales a los que se pretende aportar desde las diferentes acciones universitarias? Puede haber diferentes respuestas a esta pregunta, dependiendo del entorno social y político de cada universidad.

Sin embargo, cabe destacar que los líderes de 193 países reunidos por Naciones Unidas han acordado que el objetivo de la sociedad global es el desarrollo sostenible. La Declaración de Naciones Unidas Transformar nuestro mundo: la Agenda 2030 para el Desarrollo Sostenible (Naciones Unidas, 2015) acuerda los 17 objetivos de desarrollo sostenible (ODS) entendiéndolos como una guía para abordar los desafíos mundiales más acuciantes. Ellos representan una compleja gama de desafíos sociales, económicos y ambientales, y las universidades pueden desempeñar un rol importante en su consecución.

Por ello parece útil exponer en el BSU cómo desde la docencia, investigación, extensión y gestión universitaria se aporta al cumplimiento de los ODS o, lo que es lo mismo, dar cuenta de cómo la organización universitaria aporta al desarrollo sostenible de la sociedad en su conjunto.

La relación de los impactos universitarios con los ODS ampliaría las ventajas del balance social universitario ya que permitiría (Sustainable Development Solutions Network Australia/Pacífico, 2017):

1. Conocer e identificar lo que ya se está haciendo para contribuir al desarrollo sostenible;

2. Apropiarse de la Agenda 2030 desarrollando capacidad y liderazgo interno de los ODS;

3. Identificar prioridades, oportunidades y debilidades;

4. Integrar, implementar e incorporar los ODS en las estrategias, políticas y planes de la Universidad;

5. Monitorizar, evaluar y comunicar sus acciones con respecto a IOS ODS.

En el modelo propuesto, entonces, se toman Ios ODS como síntesis de los objetivos y valores sociales. 


\section{METODOLOGIA}

Para esta etapa del trabajo se utilizó una metodología cuantitativa. Se partió de una investigación de tipo teórico-empírica que, según Del Carril et al. (2008), parte formulando proposiciones centrales de un determinado paradigma, internándose luego en el análisis de un caso que confirma, modifica o especifica las formulaciones teóricas planteadas. Es el resultado de este análisis lo que se presenta en este trabajo.

Como métodos generales se utilizaron tanto el deductivo como el análisis y la síntesis. Como método específico se empleó la observación.

La recolección de datos se realizó a través de observación directa de normativas y documentación tanto económico-financiera como académica y entrevistas a informantes claves.

Para el desarrollo del Balance Social Universitario en una primera etapa se hizo una investigación documental de la normativa que regula el funcionamiento interno de la FCEyA, que comprende la Ordenanza №004/2013 del 20/12/2013, que aprueba el Plan de Desarrollo Institucional 2013-2023, la Ordenanza CD FCEyA №007 del 9 de setiembre de 2011, que aprueba su estructura organizacional, y la Ordenanza CD FCEyA № 002 del 22 de diciembre de 2017, que aprueba el Reglamento de Alumnos.

Además, se hicieron entrevistas con los responsables del Departamento Alumnos y la Secretaría Administrativa y se recabó información específica sobre la planta docente y no docente.

Se realizaron entrevistas al Secretario de Investigación y la Secretaria de Relaciones Institucionales para conocer sobre las líneas de investigación y extensión existentes en el 2018.

Se realizó una investigación documental de los archivos de la FCEyA, que cuentan con los convenios firmados con distintas organizaciones del medio y las actividades llevadas a cabo.

Finalmente, se procedió al relevamiento de datos relevantes de la Facultad de Ciencias Económicas y Administración en la Secretaría Económica Financiera de la Universidad Nacional de Catamarca a efectos de realizar la confección del Estado de Valores Económicos Ingresados y Distribuidos.

\section{BALANCE SOCIAL DE LA FCEYA-UNCA}

El Balance Social Universitario de la Facultad de Ciencias Económicas y de Administración de la Universidad Nacional de Catamarca ${ }^{1}$ está estructurado de la siguiente manera, conforme al mo- delo de adecuación de las normas contables argentinas que elaboró anteriormente este mismo grupo de investigación:

Presentación del Balance a cargo de la autoridad máxima de la Facultad

La presentación es breve, con la finalidad de introducir al lector en el desarrollo posterior y expresar las intenciones de la gestión con la divulgación del primer Balance Social en ámbitos de la Institución.

Es de destacar que en esta sección se presenta la justificación teórica y conceptual de relacionar los indicadores definidos en el BSU con los Objetivos de Desarrollo Sostenible.

\section{Breve historia de la Universidad}

En esta sección se presenta la historia de la FCEyA bajo un formato de línea de tiempo. Los orígenes de la FCEyA se remontan al año 1973 cuando por Resolución Rectoral №182 del 20 de septiembre, se aprueban las materias del Ciclo Básico Común y Profesional para las carreras de Contador Público Nacional y de la Licenciatura en Economía.

Seguido a ello se han resaltado los puntos más trascendentes de la historia de la Facultad que definieron el rumbo y la posición que tiene en la actualidad dentro de la comunidad educativa catamarqueña. De esta manera se pretende iniciar al lector en el conocimiento de la Casa de Estudios bajo análisis.

Datos generales de la universidad.

Esta sección tiene la finalidad de dar una idea general al lector de los propósitos institucionales y la escala de sus actividades.

Está compuesta de los siguientes subtítulos:

Estructura organizacional: se presenta la actual estructura con sus pertinentes organigramas de estructura vertical y horizontal y el Manual de Organización, donde constan las funciones de los distintos cargos de gestión, que fue aprobada mediante Ordenanza CD FCEyA №007 del 9 de setiembre de 2011. La estructura se encuentra cubierta en su totalidad salvo el cargo dependiente de la Secretaría de Posgrado: Gestión Académica de Posgrado. Esta función la cumplían los Directores de Carrera (previstos en la Estructura horizontal) en forma conjunta con el Secretario de Posgrado. 
Servicios - carreras que se dictan: actualmente se brinda a la comunidad una carrera de pre-grado: Tecnicatura en Administración de la Educación Superior; cuatro carreras de grado: Contador Público, Licenciatura en Administración, Licenciatura en Gestión de la Educación Superior y Licenciatura en Gestión Pública; y tres carreras de postgrado: Especialización en Contabilidad Superior y Auditoría, Especialización en Tributación y Maestría en Contabilidad Superior y Auditoría. Cada una de ellas se presenta en el BSU con el detalle de sus objetivos y destinatarios.

Distribución de alumnos según año de ingreso y carrera que cursa: esta información se encuentra presentada en un cuadro de doble entrada. La información abarca los años 2011 a 2018 y para las cuatro carreras de grado que representan la mayor matrícula de la FCEyA.

Líneas de Investigación: se presenta a las partes interesadas las principales líneas de investigación que posee la FCEyA. Conforme al Plan de Desarrollo Institucional (PDI) se trabaja actualmente en 4 institutos que reúnen a los investigadores por disciplinas. En estos institutos se localizan 10 proyectos de I + D acreditados por la Universidad (3 en el de Instituto de Investigaciones Contables, 1 en Economía, 1 en Estadísticas y Demografía y 5 en Administración, de los cuales 3 son del Centro de Políticas Públicas (CPP)). Se ha definido una línea estratégica de investigación y un programa interno de promoción de la investigación. Además, se hace un detalle de la cantidad de docentes investigadores categorizados en el Sistema Nacional de Incentivos.

\section{Planta Docente y No Docente}

Como una sección especial se ha decido incorporar el detalle de nuestro personal diferenciando entre la planta docente y no docente.

Para ambas categorías se presenta la información representada gráficamente de manera de facilitar su lectura indicando género y cargo que poseen. Para la categoría docente además se incluye un detalle de la dedicación y departamento al que pertenecen.

\section{Gobierno y grupos de interés}

La estructura de gobierno y conducción de la FCEyA está integrada por un Consejo Directivo, presidido por la Decana y compuesto por 7 docentes, 5 estudiantes, 1 egresado y un no docente. Todos ellos son funcionarios elegidos por todos los estamentos con un sistema de votación indirecto y representan el cogobierno de la Facultad.

La Decana, acompañada de un Vicedecano (también electo) y 4 Secretarías (Académica, Relaciones Institucionales, Posgrado e Investigación) tiene a su cargo la administración y representación legal de la Facultad. Los Secretarios son funcionarios designados por el Decano.
Los grupos de interés a los que va dirigido el Balance Social pueden ser clasificados de la siguiente manera:

Internos: se encuentra integrado por el cuerpo docente, los gremios docentes: GDU y ADUNCA; el conjunto de estudiantes, las agrupaciones estudiantiles: Franja Morada y MUPU, el Centro de Estudiantes; los no docentes, el gremio no docente: APUNCA y la misma estructura de gobierno de la FCEyA.

Externos: las autoridades de la UNCA, la Comunidad Universitaria en su conjunto, los graduados, el Consejo Profesional de Ciencias Económicas de Catamarca, las organizaciones empresariales, la CONEAU, todas las organizaciones con las que se ha firmado convenios de colaboración, las autoridades nacionales, provinciales y municipales. Y como grupo de interés externo más general la sociedad y su ambiente.

\section{Memoria de sustentabilidad}

Para la confección de la memoria de sustentabilidad se siguió el modelo adaptado de la Federación Argentina de Consejos Profesionales de Ciencias Económicas (FACPCE).

Ella incorpora el enfoque de materialidad; esto implica que más allá del amplio conjunto de asuntos relacionados con la sostenibilidad que se gestionen cotidianamente, las memorias se centrarán en asuntos críticos para el cumplimiento de sus objetivos y la administración de su impacto en la sociedad.

Se realiza un abordaje por dimensiones, definiendo cada una de ellas en función de un documento elaborado por el Consejo de Decanos de Ciencias Económicas de las Universidades Nacionales (CO.DE.C.E). Definir las dimensiones desde esta perspectiva permitirá hacer comparable la información con otras instituciones miembros del Consejo.

Para un abordaje por dimensiones, se relacionaron los indicadores elaborados con la contribución de los mismos al cumplimiento de los Objetivos de Desarrollo Sostenible definidos por la Organización de las Naciones Unidas.

En este sentido, es oportuno resaltar que al tratarse del primer periodo en que se confecciona el Balance Social, este no cuenta con datos del ejercicio anterior que puedan ser expuestos de modo comparativo.

Antes de proceder a detallar el análisis de cada una de las dimensiones resulta necesario recordar cada uno de los objetivos de desarrollo sostenible remarcando las metas de aquellos objetivos a los que contribuye la FCEyA con el desarrollo de sus actividades. 
Los objetivos y metas, según las Naciones Unidas (2015), son:

\section{1) Fin de la pobreza.}

2) Hambre cero

3) Salud y Bienestar. Metas involucradas:

a. M. 3.5: Fortalecer la prevención y el tratamiento del abuso de sustancias adictivas, incluido el uso indebido de estupefacientes y el consumo nocivo de alcohol.

4) Educación de calidad. Metas involucradas:

a. M. 4.3: De aquí a 2030, asegurar el acceso igualitario de todos los hombres y las mujeres a una formación técnica, profesional y superior de calidad, incluida la enseñanza universitaria.

b. M. 4.4: De aquí a 2030, aumentar considerablemente el número de jóvenes y adultos que tienen las competencias necesarias, en particular técnicas y profesionales, para acceder al empleo, el trabajo decente y el emprendimiento.

c. M. 4.5: De aquí a 2030, eliminar las disparidades de género en la educación y asegurar el acceso igualitario a todos los niveles de la enseñanza y la formación profesional para las personas vulnerables, incluidas las personas con discapacidad, los pueblos indígenas y los niños en situaciones de vulnerabilidad.

d. M. 4.7: De aquí a 2030, asegurar que todos los alumnos adquieran los conocimientos teóricos y prácticos necesarios para promover el desarrollo sostenible, entre otras cosas mediante la educación para el desarrollo sostenible y los estilos de vida sostenibles, los derechos humanos, la igualdad de género, la promoción de una cultura de paz y no violencia, la ciudadanía mundial y la valoración de la diversidad cultural y la contribución de la cultura al desarrollo sostenible.

5) Igualdad de Género. Metas involucradas:

a. M. 5.4: Reconocer y valorar los cuidados y el trabajo doméstico no remunerados mediante servicios públicos, infraestructuras y políticas de protección social, y promoviendo la responsabilidad compartida en el hogar y la familia, según proceda en cada país. b. M. 5.5: Asegurar la participación plena y efectiva de las mujeres y la igualdad de oportunidades de liderazgo a todos los niveles decisorios en la vida política, económica y pública.

6) Agua limpia y saneamiento. Metas involucradas:

a. M. 6.4: De aquí a 2030, aumentar considerablemente el uso eficiente de los recursos hídricos en todos los sectores y asegurar la sostenibilidad de la extracción y el abastecimiento de agua dulce para hacer frente a la escasez de agua y reducir considerablemente el número de personas que sufren falta de agua.

7) Energía asequible y no contaminante. Metas involucradas:

a. M. 7.3: De aquí a 2030, duplicar la tasa mundial de mejora de la eficiencia energética.

8) Trabajo decente y crecimiento económico. Metas involucradas:

a. M. 8.2: Lograr niveles más elevados de productividad económica mediante la diversificación, la modernización tecnológica y la innovación, entre otras cosas centrándose en los sectores con gran valor añadido y un uso intensivo de la mano de obra.

b. M. 8.3: Promover políticas orientadas al desarrollo que apoyen las actividades productivas, la creación de puestos de trabajo decentes, el emprendimiento, la creatividad y la innovación, y fomentar la formalización y el crecimiento de las microempresas y las pequeñas y medianas empresas, incluso mediante el acceso a servicios financieros.

c. M. 8.4: Mejorar progresivamente, de aquí a 2030, la producción y el consumo eficientes de los recursos mundiales y procurar desvincular el crecimiento económico de la degradación del medio ambiente, conforme al Marco Decenal de Programas sobre modalidades de Consumo y Producción Sostenibles, empezando por los países desarrollados.

d. M. 8.5: De aquí a 2030, lograr el empleo pleno y productivo y el trabajo decente para todas las mujeres y los hombres, incluidos los jóvenes y las personas con discapacidad, así como la 
igualdad de remuneración por trabajo de igual valor.

e. M. 8.7: Adoptar medidas inmediatas y eficaces para erradicar el trabajo forzoso, poner fin a las formas contemporáneas de esclavitud y la trata de personas y asegurar la prohibición y eliminación de las peores formas de trabajo infantil, incluidos el reclutamiento y la utilización de niños soldados, y, de aquí a 2025, poner fin al trabajo infantil en todas sus formas.

f. M. 8.8: Proteger los derechos laborales y promover un entorno de trabajo seguro y sin riesgos para todos los trabajadores, incluidos los trabajadores migrantes, en particular las mujeres migrantes y las personas con empleos precarios.

g. M. 8.9: De aquí a 2030, elaborar y poner en práctica politicas encaminadas a promover un turismo sostenible que cree puestos de trabajo y promueva la cultura y los productos locales.

9) Industria, innovación e infraestructura

10) Reducción de las desigualdades. Metas involucradas:

a. M. 10.2: De aquí a 2030, potenciar y promover la inclusión social, económica y política de todas las personas, independientemente de su edad, sexo, discapacidad, raza, etnia, origen, religión o situación económica u otra condición.

11) Ciudades y comunidades sostenibles:

a. M. 11.4: Redoblar los esfuerzos para proteger y salvaguardar el patrimonio cultural y natural del mundo.

12) Producción y consumo responsable. Metas involucradas:

a. M. 12.3: De aquí a 2030, reducir a la mitad el desperdicio de alimentos per cápita mundial en la venta al por menor y a nivel de los consumidores y reducir las pérdidas de alimentos en las cadenas de producción y suministro, incluidas las pérdidas posteriores a la cosecha.

b. M. 12.5: De aquí a 2030, reducir considerablemente la generación de desechos mediante actividades de prevención, reducción, reciclado y reutilización. c. M. 12.7: Promover prácticas de adquisición pública que sean sostenibles, de conformidad con las políticas y prioridades nacionales.

13) Acción por el clima

14) Vida submarina

15) Vida de ecosistemas terrestres. Metas Involucradas:

a. M. 15.9: Para 2020, integrar los valores de los ecosistemas y la diversidad biológica en la planificación nacional y local, los procesos de desarrollo, las estrategias de reducción de la pobreza y la contabilidad.

16) Paz, justicia e instituciones sólidas. Metas involucradas:

a. M. 16.3: Promover el estado de derecho en los planos nacional e internacional y garantizar la igualdad de acceso a la justicia para todos.

b. M. 16.6: Crear a todos los niveles instituciones eficaces y transparentes que rindan cuentas.

c. M. 16.7: Garantizar la adopción en todos los niveles de decisiones inclusivas, participativas y representativas que respondan a las necesidades.

17) Alianzas para lograr los objetivos.

Las dimensiones expuestas en el BSU son las siguientes:

Dimensión Contexto Institucional: La FCEyA aprueba mediante Ordenanza №004/2013 del 20/12/2013 el Plan de Desarrollo Institucional 2013-2023, en él se plantea un abordaje por ejes: UNCA.

Eje 1: Promover la reingeniería institucional de la FCEyA -

Eje 2: Contribuir a la construcción de la pertinencia y excelencia académica en la oferta de la FCEyA - UNCA en el grado y posgrado.

Eje 3: Desarrollar, producir y difundir los conocimientos científicos y tecnológicos.

Eje 4: Contribuir a mejorar la calidad de vida de la sociedad anticipándose a las demandas y problemas que se plantea en el seno de la misma. 
Cuadro 1: Análisis por Dimensiones - Dimensión

Contexto Institucional

\begin{tabular}{|c|c|c|c|c|c|c|c|}
\hline ORDEN & INDICADOR & DESCRIPCIÓN DE CONTROL & $\begin{array}{l}\text { RESULTADO } \\
\text { ACTUAL }\end{array}$ & $\begin{array}{l}\text { RESULADO } \\
\text { ANTERTOR }\end{array}$ & $\begin{array}{l}\text { MENORA } \\
\text { DETERIORO }\end{array}$ & ODS & META \\
\hline 1 & Posee misión y visión & No posee Misión y visión propia, sino que adhiere a la definida por la Universidad & No posee & no aplica & no aplica & $\begin{array}{l}\text { ODS } 4 \\
\text { ODS } 16\end{array}$ & 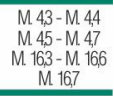 \\
\hline 2 & $\begin{array}{l}\text { PDI - Eje 1i Existe una } \\
\text { planificación global } \\
\text { aprobada por la FCEyA? }\end{array}$ & Si existe. Plan de Desarrollo Institucional 2013 - 2023 & Siposee & no aplica & no aplica & $\begin{array}{l}\text { ODS } 4 \\
\text { ODS } 16\end{array}$ & 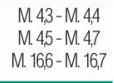 \\
\hline 3 & 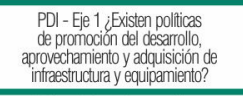 &  & Siexiste & no aplica & no aplica & $\begin{array}{l}\text { ODS } 4 \\
\text { ODS } 6 \\
\text { ODS } 7\end{array}$ & $\begin{array}{l}\text { M. } 4.3-\text { - M. } 4,2 \\
\text { M. } 6.4 \\
\text { M. } 7,3 \\
\end{array}$ \\
\hline 4 & 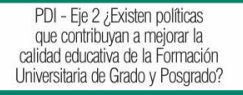 & 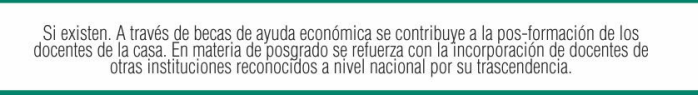 & Siexiste & no aplica & no aplica & ODS 4 & $\begin{array}{l}\text { M. } 4,3 \\
\text { M. } 4,4\end{array}$ \\
\hline 5 & $\begin{array}{l}\text { PDI - Eje } 2 \text { iExisten politicas } \\
\text { que promuevan el desarrollo } \\
\text { del a a oferta académica? }\end{array}$ & $\begin{array}{l}\text { La promoción de la oferta académica es escasa. Fundamentalamente a través de la página } \\
\text { web institucional de la fCEyA }\end{array}$ & Escaso & no aplica & no aplica & ODS 4 & M. 4,3 \\
\hline 6 & $\begin{array}{l}\text { PDI - Eje } 2 \text {, Existen polficas de } \\
\text { administracín y gestión académica } \\
\text { que promueveran la mejojar continua } \\
\text { de la calidad de los alumnos? }\end{array}$ & 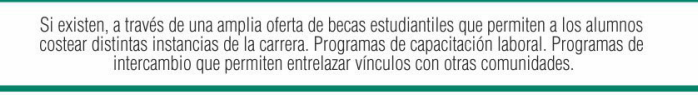 & Siexiste & no aplica & no aplica & ODS 4 & $\begin{array}{l}\text { M. } 4,3 \\
\text { M. } 4,4 \\
\text { M. } 4,6 \\
\end{array}$ \\
\hline 7 & 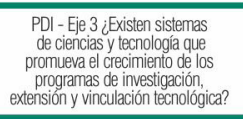 & 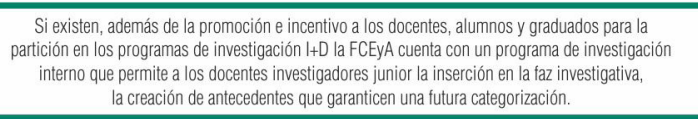 & Siexiste & no aplica & no aplica & ODS 4 & $\begin{array}{l}\text { M. } 4,3 \\
\text { M. } 4,4\end{array}$ \\
\hline 8 & 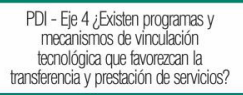 & $\begin{array}{l}\text { Si existen. La FCEEyA cuenta con } 2 \text { programas permanentes que favorecen la transferencia } \\
\text { y prestación de servicios Además de distintos Proyectos, Convenios y Jornadas } \\
\text { en donde se trabaja en esta linea. }\end{array}$ & Siexiste & no aplica & no aplica & ODS 4 & $\begin{array}{l}\text { M. } 4,3 \\
\text { M. } 4,4\end{array}$ \\
\hline 9 & $\begin{array}{l}\text { PDI- Eje } 4 \text { jexisten proyectios } \\
\text { especticos que mejoren lacapaciciad } \\
\text { de la difisisión de la producción } \\
\text { universitaria? }\end{array}$ & No posee & No posee & no aplica & no aplica & * & * \\
\hline 10 & 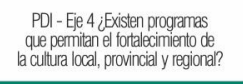 & 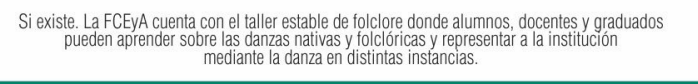 & Siposee & no aplica & по арlica & ODS 4 & M. 4,3 \\
\hline 11 & $\begin{array}{l}\text { ¿Existen programas de apoyo } \\
\text { al a aifiencia } \\
\text { acadénica de los estuduantes? }\end{array}$ & $\begin{array}{l}\text { Si existen. Mediante una amplia oferta de becas de estímulo oconómicoco y } \\
\text { capacitiación a través de la la vinculación con instituciones de la región. }\end{array}$ & Siexiste & noaplica & no aplica & ODS 4 & $\begin{array}{l}\text { M. } 4,3 \\
\text { M. } 4,4\end{array}$ \\
\hline 12 & $\begin{array}{l}\text { ¿Existen pollicicas para fomenten } \\
\text { iniciativas que promuevan una } \\
\text { mayor responsabilidad ambiental? }\end{array}$ & 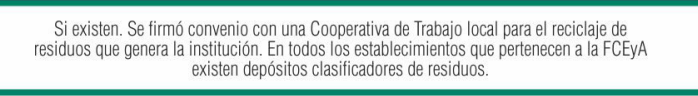 & Siexiste & no aplica & no aplica & ODS 12 & $\begin{array}{l}\text { M. } 12,5 \\
\text { M.12,7 }\end{array}$ \\
\hline 13 & 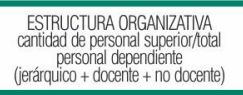 & $6 /(6+146+33)$ & $3,24 \%$ & по арlica & no aplica & $\begin{array}{l}\text { ODS } 5 \\
\text { ODS } 8 \\
\text { ODS } 10\end{array}$ & $\begin{array}{l}\text { M. } 5,5 \\
\text { M. } 8,8 \\
\text { M. } 10,2\end{array}$ \\
\hline 14 & $\begin{array}{l}\text { ESTRUCTURA ORGANZATIVA } \\
\text { cantidad de personal docenteflotol } \\
\text { personal dependiente } \\
\text { (jerarquico + docente + no docente) }\end{array}$ & $146 /(6+146+33)$ & $78,92 \%$ & no aplica & no aplica & $\begin{array}{c}\text { ODS } 5 \\
\text { ODS } 8 \\
\text { ODS } 10 \\
\end{array}$ & $\begin{array}{r}\text { M. } 5,5 \\
\text { M. } 8,8 \\
\text { M. } 10,2 \\
\end{array}$ \\
\hline 15 & 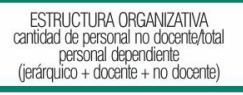 & $33 /(6+146+33)$ & $17,84 \%$ & no aplica & no aplica & $\begin{array}{l}\text { ODS } 5 \\
\text { ODS } 8 \\
\text { ODS } 10 \\
\end{array}$ & $\begin{array}{l}\text { M. } 5,5 \\
\text { M. } 8,8 \\
\text { M. } 10,2 \\
\end{array}$ \\
\hline 16 & $\begin{array}{l}\text { ¿Existen planes de evaluación } \\
\text { para total de docentes? }\end{array}$ & 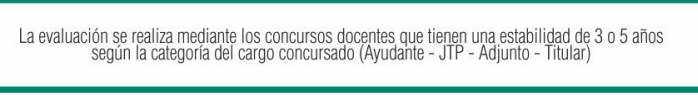 & Siexiste & no aplica & no aplica & $\begin{array}{l}00 S 4 \\
0058\end{array}$ & $\begin{array}{l}\text { M. } 4,3 \\
\text { M. } 4,4 \\
\text { M. } 8,8 \\
\end{array}$ \\
\hline 17 & $\begin{array}{l}\text { ¿Existen planes de vevaluacín } \\
\text { para tolala des no docentes? }\end{array}$ & 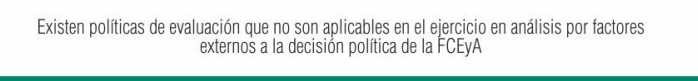 & No se aplican & no aplica & no aplica & $\begin{array}{l}\text { ODS } 4 \\
\text { ODS } 8\end{array}$ & $\begin{array}{l}\text { M. } 4,3 \\
\text { M. } 8,8\end{array}$ \\
\hline 18 & $\begin{array}{l}\text { ¿Existen politicas de admisión, } \\
\text { seguimimento y permanencia' } \\
\text { de alumnos? }\end{array}$ & $\begin{array}{l}\text { Si existen pollicicas de admisión a la FCEEA por reglamento de alumnos definiendo el mismo } \\
\text { como irrestricto. La polfitica de seguimiento y permanencia es inexistentie. }\end{array}$ & Aplicación Parcial & no aplica & no aplica & ODS 4 & $\begin{array}{l}\text { M. } 4,3 \\
\text { M. } 4,5\end{array}$ \\
\hline 19 & 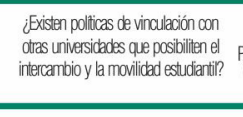 & 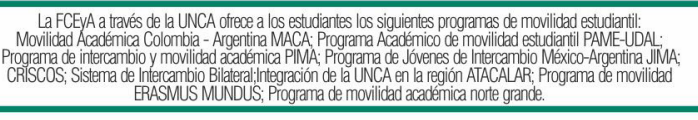 & Siposee & no aplica & no aplica & ODS 4 & $\begin{array}{l}\text { M. } 4,3 \\
\text { M. } 4,4 \\
\text { M. } 4, b\end{array}$ \\
\hline 20 & $\begin{array}{l}\text { ¿Existen mecanismos de } \\
\text { seguimientio sobre los gradadados? }\end{array}$ & Si existen, aprobados mediante Ordenanza CD № 009/2018. & Siexisten & no aplica & no aplica & ODS 4 & $\begin{array}{l}\text { M. } 4,3 \\
\text { M. } 4,4\end{array}$ \\
\hline 21 & $\begin{array}{l}\text { EExisten planes de } \\
\text { auto-evaluación periódica? }\end{array}$ & 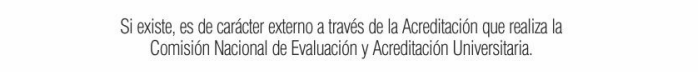 & Siexisten & no aplica & по aplica & ODS 4 & $\begin{array}{l}\text { M. } 4,3 \\
\text { M. } 4,4\end{array}$ \\
\hline 22 & 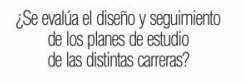 & Si se evalúa,a a través del comité interdepartamental integrado por los Jefess de Departamentios. & Siexisten & по арlica & по арlica & ODS 4 & $\begin{array}{l}\text { M. } 4,3 \\
\text { M. } 4,4\end{array}$ \\
\hline
\end{tabular}


Dimensión Docencia: para la elaboración de estos indicadores se tomó en consideración las carreras de pregrado, grado y postgrado que se dictan, la planta docente y la cantidad de alumnos de la FCEyA al año 2018.

\section{Cuadro 2: Análisis por Dimensiones - Dimensión Docencia}

\begin{tabular}{|c|c|c|c|c|c|c|c|}
\hline ORDEN & INDICADOR & DESCRIPCIÓN DE CONTROL & $\begin{array}{l}\text { RESULADO } \\
\text { ACTUAL }\end{array}$ & $\begin{array}{l}\text { RESULTADO } \\
\text { ANTERTOR }\end{array}$ & $\begin{array}{l}\text { MENORA } \\
\text { DEIERTOORO }\end{array}$ & ODS & META \\
\hline 1 & $\begin{array}{l}\text { Cantidad de carreras de pre-grado, } \\
\text { grado y pos-grado. }\end{array}$ & 9 & 9 & No aplica & No aplica & OSD 4 & M. 4,3 \\
\hline 2 & $\begin{array}{l}\text { Carreras de grado: } \\
\text { Asignaturas con formación en } \\
\text { valores/ total asignaturas por carrera }\end{array}$ & $\begin{array}{l}\text { total materias }=94 \\
\text { Mat. For. en Val. }=7\end{array}$ & $7,45 \%$ & № aplica & № aplica & $\begin{array}{l}\text { ODS } 8 \\
\text { ODS } 12\end{array}$ & $\begin{array}{l}\text { M. } 8,3-\text { M. } 8.8 \\
\text { M. } 8,5-1.8 .87 \\
\text { M. } 12,3 \\
\text { M. } 12,9\end{array}$ \\
\hline 3 & $\begin{array}{l}\text { Docentes por } \\
\text { concurso/total docentes }\end{array}$ & $\begin{array}{l}\text { total doc: } 146 \\
\text { doc x conc:106 }\end{array}$ & $72,60 \%$ & № aplica & № aplica & $\begin{array}{l}\text { ODS } 8 \\
\text { ODS } 12\end{array}$ & $\begin{array}{l}\text { M. } 8.3-\text { M. } .8 .4 \\
\text { M. } 8,5-1.8 .7 \\
\text { M. } 12.3 \\
\text { M. } 12,9\end{array}$ \\
\hline 4 & $\begin{array}{l}\text { Docentes } \\
\text { posgraduados/Total docentes }\end{array}$ & $\begin{array}{l}\text { doc.pos: } 58 \\
\text { total: } 146\end{array}$ & $39,73 \%$ & № aplica & No aplica. & $\begin{array}{l}\text { ODS } 4 \\
\text { ODS } 8 \\
\text { ODS } 12\end{array}$ & $\begin{array}{c}M 45 \\
M 83-M 84 \\
M 85-M 87 \\
M 123-M 129\end{array}$ \\
\hline 5 & $\begin{array}{l}\text { Estudiantes colaborando con } \\
\text { docentes/total docentes }\end{array}$ & $\begin{array}{l}\text { total doce: } 146 \\
\text { est.: } 13\end{array}$ & 0,09\% & No aplica & No aplica & $\begin{array}{l}\text { ODS } 4 \\
\text { ODS } 8 \\
\text { ODS } 12\end{array}$ & $\begin{array}{c}\text { M 45 } \\
\text { M 83-M } 84 \\
\text { M 85- M } 87 \\
\text { M 123-M. 129 }\end{array}$ \\
\hline 6 & $\begin{array}{l}\text { Cantidad alumnos } \\
\text { graduados / total alumnos }\end{array}$ & $\begin{array}{l}\text { total alum: } 1439 \\
\text { grad: } 65\end{array}$ & $0,05 \%$ & No aplica & № aplica & $\begin{array}{l}\text { ODS } 4 \\
\text { ODS } 8 \\
\text { ODS } 12\end{array}$ & $\begin{array}{c}M 45 \\
M .83-M .84 \\
\text { M } 52-M .87 \\
M 123-M .129\end{array}$ \\
\hline 7 & $\begin{array}{l}\text { Cantidad alumnos } \\
\text { graduados/ alumnos ingresantes }\end{array}$ & $\begin{array}{l}\text { ing. } 211 \\
\text { grad: } 65\end{array}$ & $30,81 \%$ & No aplica & No aplica & $\begin{array}{l}\text { ODS } 8 \\
\text { ODS } 12\end{array}$ & $\begin{array}{l}\text { M 83-M } 84 \\
\text { M 85-M 87 } \\
\text { M 123-M 129 }\end{array}$ \\
\hline 8 & $\begin{array}{l}\text { Cantidad alumnos con } \\
\text { prácticas/ total alumnos activos. }\end{array}$ & $\begin{array}{l}\text { Alum pract: } 20 \\
\text { Alumnos: } 1439\end{array}$ & $1,39 \%$ & № aplica & No aplica & $\begin{array}{l}\text { ODS } 8 \\
\text { ODS } 12\end{array}$ & $\begin{array}{l}\text { M 83-M } 84 \\
\text { M 85-M 87 } \\
M \text { M 123-M 129 }\end{array}$ \\
\hline 9 & $\begin{array}{l}\text { Cantidad de organizzaciones donde } \\
\text { los alumnos realizan prácticas. }\end{array}$ & 27 & 27 & No aplica & No aplica & $\begin{array}{l}\text { ODS } 4 \\
\text { ODS } 8 \\
\text { ODS } 12\end{array}$ & $\begin{array}{c}\text { M.45 } \\
\text { M.83-M } 84 \\
\text { M } 85-M 87 \\
\text { M. 123-M 129 }\end{array}$ \\
\hline
\end{tabular}

Fuente: Facultad de Ciencias Económicas y de Administración de la Universidad Nacional de Catamarca (2018)
Dimensión Investigación: para la elaboración de estos indicadores se tomó en consideración entre otros la planta docente total, la cantidad de docentes investigadores y el total de alumnos que integran proyectos de investigación. 
Cuadro 3: Análisis por Dimensiones - Dimensión Investigación

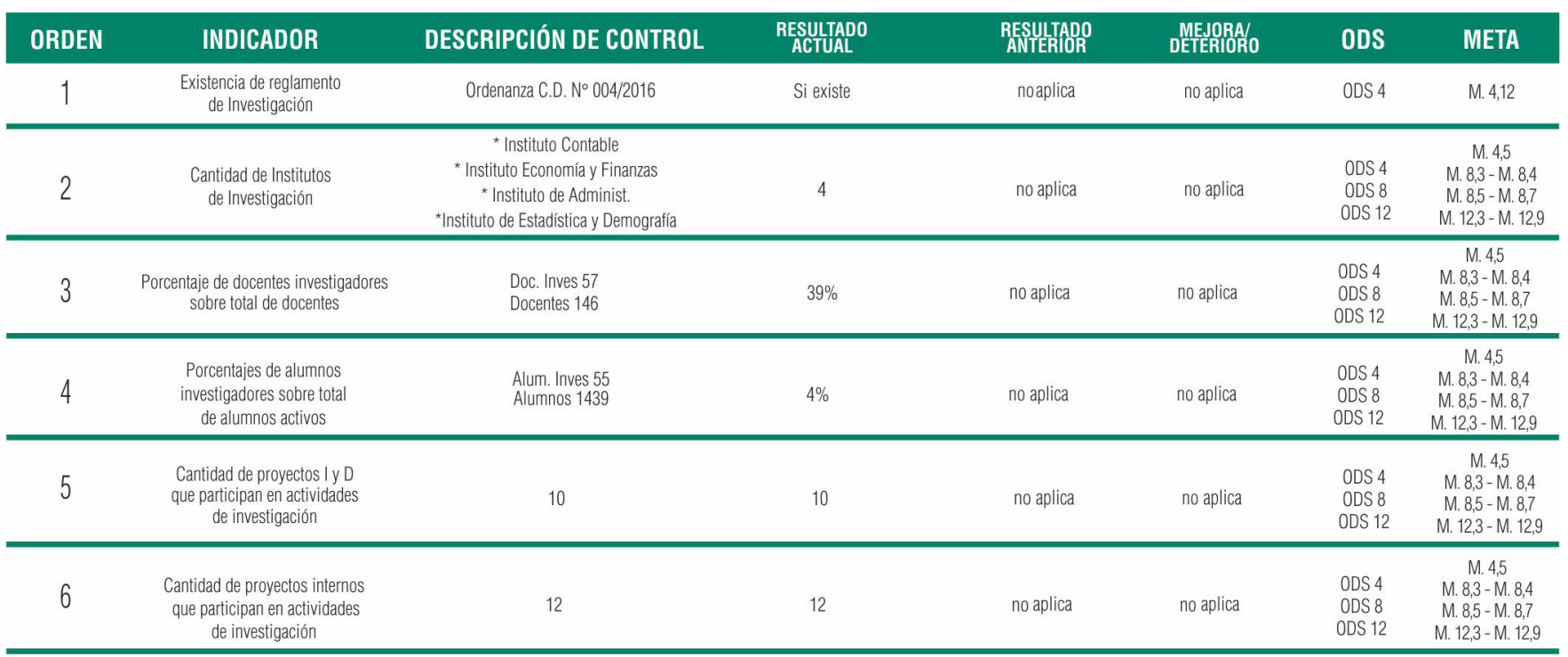

Fuente: Facultad de Ciencias Económicas y de Administración de la Universidad Nacional de Catamarca (2018)

Dimensión Extensión: para la elaboración de estos indicadores se tomó en consideración entre otros la planta docente total, la cantidad de docentes extensionistas y el total de alumnos que integran proyectos de extensión.

Cuadro 4: Análisis por Dimensiones - Dimensión Extensión

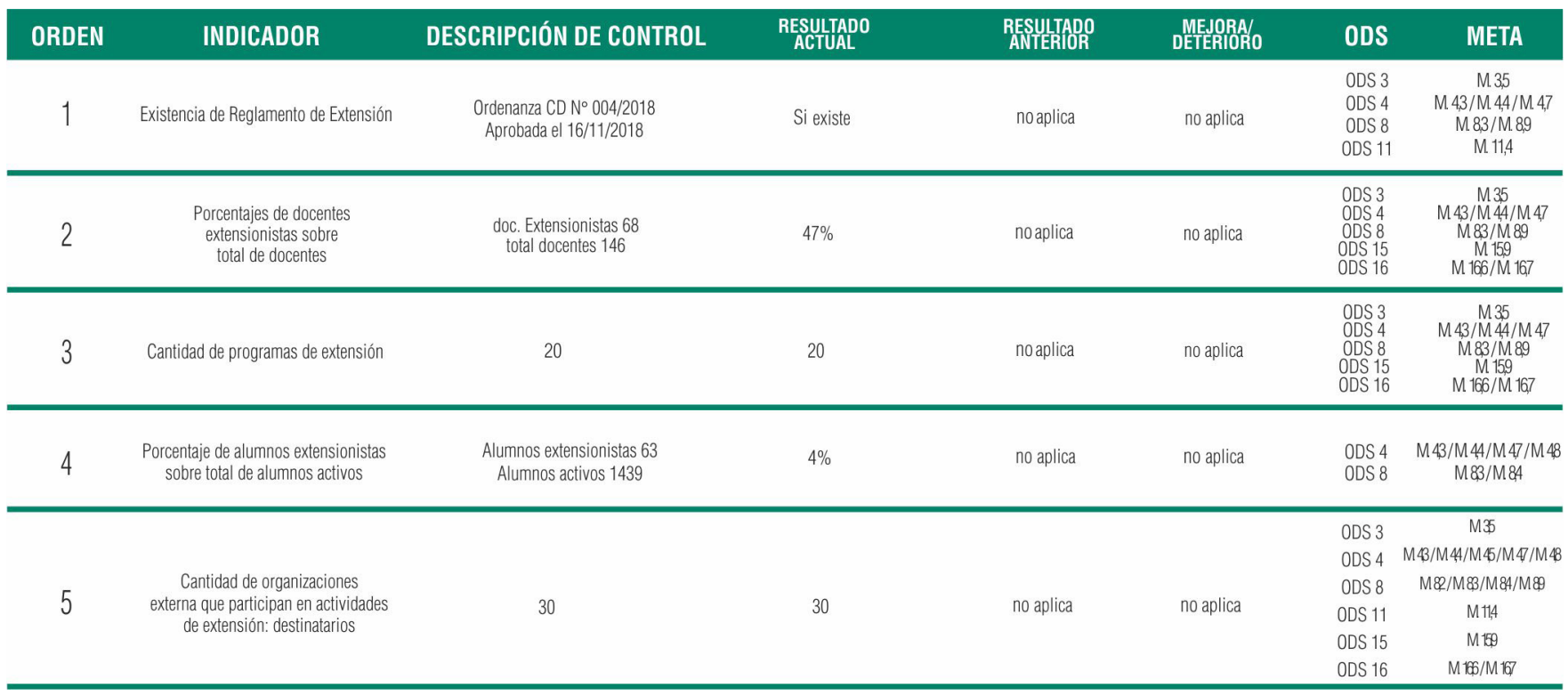




\section{Estado de Valores Económicos Ingresados y distribuidos}

El Estado de Valores Económicos Ingresados y Distribuidos (EVEID) está diseñado en base a la idea del Estado de Valores Económicos Generados y Distribuidos de las Resoluciones Técnicas № 36 y 44 de la Federación Argentina de Consejos Profesionales en Ciencias Económicas.

El presente EVEID muestra valores ingresados, ya que la generación de recursos propios tiene poca importancia relativa con respecto a los aportes del Tesoro Nacional.

Las cifras se relacionan con el segmento de la Contabilidad Gubernamental, más específicamente la Contabilidad de Presupuesto.

Se puede observar a la izquierda los recursos ingresados en relación a las diferentes fuentes de financiamiento a las que accedió la FCEyA en el ejercicio económico 2018. A la derecha los gastos ejecutados. En relación a los gastos ejecutados puede leerse en los totales de las filas los gastos por grupos de interés y en los totales de columnas los gastos de cada dimensión definida para el trabajo. Este EVEID está expresado en moneda argentina, valores ejecutados 2018.

El total de fondos ingresados se distribuye según fuentes de financiamiento, dejando en evidenciael impacto de los aportes del Tesoro Nacional (89\%).

La Distribución de los Ingresos, según las dimensiones definidas corresponde a: Docencia (67\%), Investigación y Transferencia (14\%), Gestión (11\%) y Extensión y Vinculación (8\%).

Cuadro 5: Estado de Valor Económico Ingresado y Distribuido (en miles de pesos)- Periodo 2018
Los Ingresos según Actividades Sustantivas se distribuyeron en:

\author{
Personal Superior (5\%) \\ Personal Docente (41\%) \\ Personal No Docente (16\%) \\ Alumnos (1\%) \\ Prov. de Bienes de Consumo (1\%) \\ Prov. de Servicios (2\%) \\ Prov. de Bs. de Capital - Obra (7\%)
}

El modelo del EVEID es comparativo y, al ser el primer ejercicio presentado, no tiene resultados de ejercicios anteriores.

El desarrollo del Proyecto permitió analizar y reflexionar sobre la Responsabilidad Social Universitaria (RSU), el estado actual y su proyección al futuro.

El desarrollo del trabajo permitió analizar y reflexionar sobre la Responsabilidad Social Universitaria (RSU) y la relevancia de contar con un sistema informativo contable como base para la realización de un balance social universitario que optimice la gestión académica y socio comunitaria de la Facultad de Ciencias Económicas y de Administración.

Finalmente, podemos decir que este balance permitió valorar la formación de profesionales comprometidos con valores y principios de sensibilidad humana, social y ecológica al servicio de su comunidad lo cual indica el alto impacto en la formación de los alumnos y en la gestión académica por lo cual es altamente positivo considerar su continuidad.

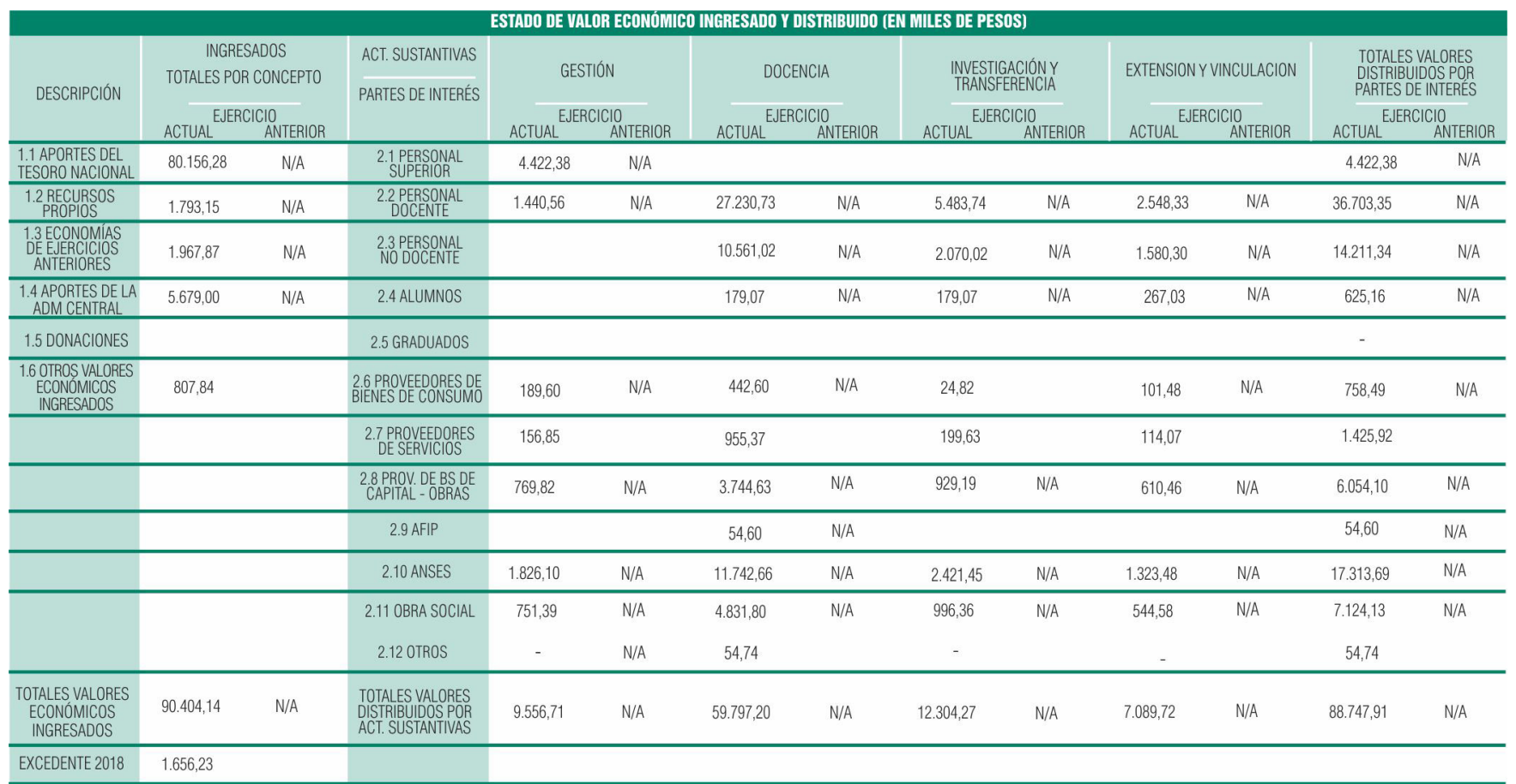




\section{CONCLUSIONES}

El trabajo de investigación permitió analizar y reflexionar acerca de la importancia que tiene la disciplina contable para confeccionar, describir, interpretar e informar, a través de un balance social universitario, el impacto de las actividades universitarias propuestas.

Un aspecto muy importante de las universidades es la autonomía institucional; esta autonomía debe ser entendida y desarrollada dentro de un marco constante de actividades y decisiones éticas que se visibilicen en informes de rendiciones de cuentas hacia la sociedad, vinculada con el concepto de Accountability, el cual se reconoce como un aspecto central de la responsabilidad social (UNESCO, 1998, p. 8). En este sentido, la autonomía se orienta a que los equipos de gestión de las diferentes universidades a nivel mundial puedan desarrollar una buena administración especialmente en la generación de recursos debido a la disminución constante de los recursos provenientes del Estado para la educación superior.

El desarrollo del Proyecto permitió analizar y reflexionar sobre la Responsabilidad Social Universitaria (RSU), el estado actual, su proyección al futuro y la relevancia de contar con un sistema informativo contable que incluya información no financiera.

Puede entonces resumirse las siguientes conclusiones:

La disciplina contable cuenta con elementos suficientes para confeccionar balances sociales para todo tipo de organizaciones, incluidas las universidades resultando posible y ventajoso confeccionar BSU confiables y complementarios de los estados financieros.

Teniendo en cuenta los avances en el reconocimiento de los impactos sociales y ambientales para lograr junto a los impactos económicos el tan ansiado desarrollo sostenible, la teoría contable debe avanzar hacia el reconocimiento absoluto de la existencia de otros segmentos además del de Contabilidad Financiera, dando más fuerza a las investigaciones y normas profesionales existentes al respecto.

Los segmentos de la Contabilidad Social y Ambiental 0 información no financiera como la llaman en Europa, adquieren en el mundo una importancia creciente. Los sistemas de información contable deben ampliarse con información social, no financiera útil a los segmentos de Contabilidad Social y Ambiental.

Los grupos de interés de las universidades pueden identificarse claramente y beneficiarse con información que facilite su participación en las decisiones sobre el devenir universitario.
Finalmente, es destacable que este balance permitió valorar la formación de profesionales comprometidos con valores y principios de sensibilidad humana, social y ecológica al servicio de su comunidad, lo cual indica el alto impacto en la formación de Ios alumnos y en la gestión académica, por lo cual es altamente positivo considerar su continuidad.

\section{REFERENCIAS}

Del Carril, J. C., De Marco, M. y Usandivaras, S. (2008). Técnicas para construir y exponer temas económicos y administrativos (2a ed.). Facultad de Ciencias Económicas de la Universidad Nacional de Tucumán.

Facultad de Ciencias Económicas y de Administración de la Universidad Nacional de Catamarca. (2018). Balance Social. Correspondiente al Ejercicio Económico 2018. http://eco.unca.edu.ar/docs/ balance2018.pdf

Gaete Quezada, R. (2017). Responsabilidad Social Universitaria: una nueva mirada a la relación de la universidad con la sociedad desde la perspectiva de las partes interesadas. Un estudio de caso [Tesis de doctorado, Universidad de Valladolid]. http://www.beu. extension.unicen.edu.ar/xmlui/bitstream/handle/123456789/232/ RESPONSABILIDAD\%20SOCIAL\%20UNIVERSITARIA\%20UNA. pdf?sequence=1\&isAllowed=y

Naciones Unidas (2015). Resolución aprobada por la Asamblea General del 25 de septiembre de 2015. https://unctad.org/system/ files/official-document/ares70d1_es.pdf.

Resolución Técnica №36 de 2013 [Federación Argentina de Consejos Profesionales en Ciencias Económicas]. Normas Contables Profesionales: Balance Social.

Sustainable Development Solutions Network Australia/Pacífico. (2017). Cómo empezar con los ODS en las universidades. Una guía para las universidades, los centros de educación superior y el sector Académico (edición en español). https://www.utn.edu.ar/ images/Secretarias/SGral/Integracion/GuiaUNiversidadesODS.pdf

Vallaeys, F. (2006). Breve marco teórico de responsabilidad social universitaria. En F. Vallaeys y L. Carrizo, Responsabilidad Social Universitaria. Banco Interamericano de desarrollo. https://www. uv.mx/APPS/CU0/TALLERRSU/Docs. \%20RSU\%20para \%20 INEGI/Breve\%20marco\%20te\%C3\%B3rico\%20de\%20Responsabilidad\%20Social\%20Universitaria.pdf

UNESCO (1998). Autonomía, responsabilidad social y libertad académica. VII Conferencia Mundial de Educación. http://unesdoc. unesco.org/images/0011/001135/113549so.pdf 